\title{
Incidence and Factors of Prolonged Postoperative Ileus in Gastric Cancer Surgery
}

\author{
Wang Ning, Chen Runkai, Cui Hao and Zheng Yiqiong* \\ Department of General Surgery, General Hospital of PLA, Beijing 100853, China \\ ${ }^{\star}$ Corresponding author: Zheng Yiqiong, Department of General Surgery, General Hospital of PLA, Beijing 100853, China
}

Received: January 14, 2021; Accepted: February 01, 2021; Published: February 03, 2021

\begin{abstract}
Objective: Prolonged Postoperative Ileus (PPOI) is a common complication after abdominal surgery, but data about incidence and risk factors of PPOI for patients with gastric cancer are rare. We sought to investigate the incidence and related incidental factors of PPOI.

Methods: A retrospective cohort study was carried out using a registry database consecutively collected from June 2016 to October 2016 . The incidence and incidental factors of PPOI after gastric cancer surgery were calculated and analyzed.

Results: There were 22 patients diagnosed with PPOI. The incidence of PPOI after gastric cancer surgery was $26.5 \%$. There were significant differences in the PPOI among ages, postoperative body temperature, postoperative opioid agents use (Dezocine) $(\mathrm{P}<0.05)$. Logistic regression analysis results showed that the age $\geq 65$ years, postoperative temperature $\geq 38^{\circ} \mathrm{C}$, the use of Dezocine after surgery were the independent risk factors of PPOI after gastric cancer surgery.
\end{abstract}

Conclusion: The occurrence of PPOI after gastric cancer surgery has great relationship with age, postoperative temperature, the use of Dezocine after surgery. We may accelerate the course of convalescence by strengthening the management of perioperative periodandtaking reasonable measures to against the risk factors.

Keywords: Gastric cancer, Prolongedpostoperative ileus, Incidental factor

Prolonged Post-Operative Ileus (PPOI) is an aberrant pattern of gastrointestinal motility, most frequently occurring after abdominal surgery. The clinical manifestations include abdominal pain, nausea, vomiting, moderate to severe sick, intolerable of a solid diet and a delayed passage of flatus and stool, which usually resolves spontaneously within 2 to 3 days [1,2]. If the symptoms persist for more than 4 days, Prolonged Postoperative Ileus (PPOI) is defined [3]. PPOI hampers the patients' recovery, increases postoperative morbidity and leads to longer length of hospital stay [4]. Understanding the incidence and factors of PPOI can help clinicians take effective measures to reduce the incidence of PPOI, and ultimately achieve rapid recovery of patients. This study retrospectively collected the clinical information of gastric cancer patients who received surgical treatment in our department, analyzed the related factors of PPOI occurrence, in order to take targeted prevention and treatment measures.

\section{Materials and Methods}

\section{Study Population}

A retrospective cohort study was carried out using a PPOI registry database consecutively collected between June 2016 and October 2016 in Chinese PLA General Hospital. Among them, 62 were male and 21 were female, the ratio of male to female was about 3:1, they were between 39 and 89 years old, and the average age was (60.1 \pm
11.0) years. Among them, 34 cases underwent total gastrectomy, 40 cases underwent distal gastrectomy and 9 cases underwent proximal gastrectomy.

\section{Diagnosis of PPOI}

The definition of PPOI was adopted from the results of a systematic review and global survey [3]. The validity of this concept was universally accepted by a variety of investigators [5-8]. Accordingly, diagnoses of PPOI were identified if two or more of following events after day 4 postoperatively: (a), nausea or vomiting; (b) inability to tolerate an oral diet over the prior 24 hour; (c) absence of flatus over the prior 24 hours; (d) abdominal distension; (e) radiologic confirmation.

\section{Method}

The clinical data of 83 patients with gastric cancer were collected, including gender, age, BMI index, previous abdominal surgery history, surgical method, surgical resection range, abdominal incision length, operation time, intraoperative blood loss, postoperative body temperature, postoperative serum leukocyte level, preoperative serum albumin level, postoperative serum albumin level, preoperative serum $\mathrm{K}+$ level, postoperative blood loss, the level of serum $\mathrm{K}+$, the use of dezocine after operation, perioperative blood transfusion, the first time to get out of bed after operation and pathological stage. Objective to analyze the influence of various factors on the occurrence of PPOI. 


\section{Statistical Analysis}

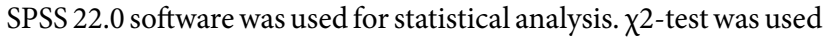
for count data. Logistic regression model was used for independent factor analysis. $\mathrm{P}<0.05$ was considered as statistical significance.

\section{Results}

\section{Postoperative Complications of PPOI}

There were 30 patients who did not exhaust and defecate within 96 hours after gastric operation, 14 patients with moderate to severe nausea and vomiting, and 28 patients with moderate to severe abdominal distension. According to the diagnostic criteria, 22 cases of PPOI occurred in 83 patients (including 3 cases of non-exhaust defecation combined with moderate to severe nausea and vomiting within $96 \mathrm{~h}, 15$ cases of non-exhaust defecation combined with moderate to severe abdominal distension within $96 \mathrm{~h}$, and 4 cases of non-exhaust defecation combined with moderate to severe nausea, vomiting and moderate to severe abdominal distension within 96h), with an incidence of $26.5 \%$. After conservative treatment, the clinical symptoms of 22 patients with PPOI were improved.

\section{Relationship between PPOI and Clinical Factors}

Univariate analysis showed that age $(\mathrm{P}=0.001)$, postoperative body temperature $(\mathrm{P}=0.031)$, postoperative serum $\mathrm{K}+$ level $(\mathrm{P}=$ $0.017)$ and postoperative analgesia with dezocine $(\mathrm{P}=0.014)$ were significantly associated with PPOI. See Table 1.

The results showed that age $\geq 65$ years old, postoperative body temperature $\geq 38^{\circ} \mathrm{C}$, postoperative use of dezocine analgesia were the independent risk factors of PPOI in patients after gastric surgery. See Table 2.

Table 1: The results of the univariate analysis for factors related to PPOI.

\begin{tabular}{|c|c|c|c|c|}
\hline Subgroup & Number of study's & PPOI [例 (\%)] & $\chi^{2}$ Value & P Value \\
\hline \multicolumn{5}{|l|}{ Sex } \\
\hline Male & 62 & $17(27.4)$ & \multirow{2}{*}{0.105} & \multirow{2}{*}{0.746} \\
\hline Female & 21 & $5(23.8)$ & & \\
\hline \multicolumn{5}{|l|}{ Age (years) } \\
\hline$\geq 65$ & 26 & $13(50.0)$ & \multirow{2}{*}{10.727} & \multirow{2}{*}{0.001} \\
\hline$<65$ & 57 & $9(15.8)$ & & \\
\hline \multicolumn{5}{|l|}{ BMI $\left(\mathrm{kg} / \mathrm{m}^{2}\right)$} \\
\hline$\geq 24$ & 49 & $12(24.5)$ & \multirow{2}{*}{0.250} & \multirow{2}{*}{0.617} \\
\hline$<24$ & 34 & $10(29.4)$ & & \\
\hline \multicolumn{5}{|l|}{$\begin{array}{c}\text { Previous abdominal } \\
\text { surgery }\end{array}$} \\
\hline Yes & 16 & $4(25.0)$ & \multirow{2}{*}{0.023} & \multirow{2}{*}{0.879} \\
\hline No & 67 & $18(26.9)$ & & \\
\hline \multicolumn{5}{|l|}{ Operation methods } \\
\hline Open surgery & 31 & $10(32.3)$ & \multirow[b]{2}{*}{0.840} & \multirow[b]{2}{*}{0.359} \\
\hline $\begin{array}{l}\text { Laparoscopic } \\
\text { surgery }\end{array}$ & 52 & $12(23.1)$ & & \\
\hline $\begin{array}{c}\text { Surgical resection } \\
\text { range }\end{array}$ & & & & \\
\hline
\end{tabular}

\begin{tabular}{|c|c|c|c|c|}
\hline Total stomach & 34 & $10(29.4)$ & \multirow{3}{*}{0.279} & \multirow{3}{*}{0.870} \\
\hline Distal stomach & 40 & $10(25.0)$ & & \\
\hline Proximal stomach & 9 & $2(22.2)$ & & \\
\hline \multicolumn{5}{|l|}{$\begin{array}{l}\text { Length of abdominal } \\
\text { incision }(\mathrm{cm})\end{array}$} \\
\hline$>10$ & 34 & $9(26.5)$ & \multirow{2}{*}{0} & \multirow{2}{*}{0.995} \\
\hline$\leq 10$ & 49 & $13(26.5)$ & & \\
\hline \multicolumn{5}{|l|}{ Operation time (h) } \\
\hline$\geq 4$ & 39 & $9(23.1)$ & \multirow{2}{*}{0.444} & \multirow{2}{*}{0.505} \\
\hline$<4$ & 44 & $13(29.5)$ & & \\
\hline \multicolumn{5}{|l|}{$\begin{array}{l}\text { Operative blood loss } \\
(\mathrm{ml})\end{array}$} \\
\hline$\geq 200$ & 39 & $7(17.9)$ & \multirow{2}{*}{2.765} & \multirow{2}{*}{0.096} \\
\hline$<200$ & 44 & $15(34.1)$ & & \\
\hline \multicolumn{5}{|l|}{$\begin{array}{c}\text { Postoperative body } \\
\text { temp }\left({ }^{\circ} \mathrm{C}\right)\end{array}$} \\
\hline$\geq 38.0$ & 17 & $8(47.1)$ & \multirow{2}{*}{4.636} & \multirow{2}{*}{0.031} \\
\hline$<38.0$ & 66 & $14(21.2)$ & & \\
\hline \multicolumn{5}{|l|}{$\begin{array}{c}\text { Postoperative WBC } \\
\left(\times 10^{9} / \mathrm{L}\right)\end{array}$} \\
\hline$\geq 10$ & 66 & $17(25.8)$ & \multirow{2}{*}{0.093} & \multirow{2}{*}{0.761} \\
\hline$<10$ & 17 & $5(29.4)$ & & \\
\hline \multicolumn{5}{|l|}{$\begin{array}{c}\text { Preoperative albumin } \\
(\mathrm{g} / \mathrm{L})\end{array}$} \\
\hline$\geq 30$ & 82 & $21(25.6)$ & \multirow{2}{*}{2.807} & \multirow{2}{*}{0.094} \\
\hline$<30$ & 1 & $1(100.0)$ & & \\
\hline \multicolumn{5}{|l|}{$\begin{array}{l}\text { Postoperative } \\
\text { albumin }(\mathrm{g} / \mathrm{L})\end{array}$} \\
\hline$\geq 30$ & 62 & $14(22.6)$ & \multirow{2}{*}{1.938} & \multirow{2}{*}{0.164} \\
\hline$<30$ & 21 & $8(38.1)$ & & \\
\hline \multicolumn{5}{|l|}{$\begin{array}{c}\text { Preoperative } \mathrm{K}^{+} \\
(\mathrm{mmol} / \mathrm{L})\end{array}$} \\
\hline$\geq 3.0$ & 83 & $22(26.5)$ & \multirow{2}{*}{-} & \multirow{2}{*}{-} \\
\hline$<3.0$ & 0 & 0 & & \\
\hline $\begin{array}{l}\text { Postoperative K+ } \\
(\mathrm{mmol} / \mathrm{L})\end{array}$ & & & & \\
\hline$\geq 3.0$ & 81 & $20(24.7)$ & -60 & 2017 \\
\hline$<3.0$ & 2 & $2(100)$ & 0.002 & \\
\hline $\begin{array}{l}\text { Postoperative } \\
\text { analgesia with } \\
\text { Dezuocine }\end{array}$ & & & & \\
\hline Yes & 38 & $15(39.5)$ & $10-0$ & 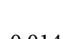 \\
\hline No & 45 & $7(15.6)$ & & \\
\hline Blood transfusion & & & & \\
\hline Yes & 22 & $7(31.8)$ & 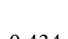 & $0-1$ \\
\hline No & 61 & $15(24.6)$ & 0.404 & 0.010 \\
\hline $\begin{array}{c}\text { Postoperative } \\
\text { ambulation time }(\mathrm{h})\end{array}$ & & & & \\
\hline$\geq 24$ & 47 & $15(31.9)$ & & 20 \\
\hline$<24$ & 36 & $7(19.4)$ & 1.027 & \\
\hline $\begin{array}{c}\text { Postoperative tumor } \\
\text { stage }\end{array}$ & & & & \\
\hline III $^{\sim}$ IV & 41 & $13(31.7)$ & & \\
\hline $\mathrm{I}^{\sim} \mathrm{II}$ & 42 & $9(21.4)$ & 1.125 & 0.289 \\
\hline
\end{tabular}


Table 2: The results of the multivariable logistic analysis for factors related to PPOI.

\begin{tabular}{|c|c|c|c|c|c|}
\hline & B & SE & Wald & P值 & OR $\%$ CI \\
\hline Age $($ years $) \geq 65$ & 2.857 & 1.177 & 5.895 & 0.015 & $0.3505-0.6495$ \\
\hline Postoperative body temp $\left({ }^{\circ} \mathrm{C}\right) \geq 38$ & 2.764 & 1.110 & 6.202 & 0.013 & 17.415 \\
\hline Postoperative analgesia with Dezuocine & 3.062 & 1.189 & 6.631 & $0.2066-0.7334$ \\
\hline
\end{tabular}

\section{Discussion}

Ambiguity surrounding the definition of PPOI has obscured the ability to accurately determine its incidence, although studies have typically placed this at between $10-25 \%$ following major elective abdominal surgery $[9,10]$. In our study, the incidence of PPOI after gastric surgery was $26.5 \%$, which was lower to that reported by Huang et al., (32.4\%) in gastric cancer [6]. The incidence of PPOI was variable in different studies due to the ambiguity about the definition. Controversies have mainly focused on the duration of ileus that should be regarded as prolonged. An observational study of 2400 consecutive patients determined 3 days as prolonged ileus, whereas Dai et al., specified 4 days and Artinyan et al., defined more than 6 days $[5,11,12]$. A global survey and systematic review extracted definitions from 52 identified trials and proposed 4 days as a standardized endpoint for PPOI [2]. It was well accepted in subsequent studies, and we also adopted this definition as diagnostic criteria in our study $[4,13]$. Given the variability in the definitions of this significant complication, further research is necessary to establish a more precise, validated definition.

Advanced age ( $>65$ years) was identified as an independent risk factor for PPOI in our study, consistent with the finding of several previous studies [6,14]. A previous mechanism research has demonstrated that imbalances between pro- and anti-inflammatory mechanisms may be the underlying pathophysiology for the increased susceptibility to POI and the increased severity and duration of POI observed in the elderly. Moreover, elderly patients generally have a decreased nutritional and functional condition, as reflected by a higher NRS 2002 score and a higher prevalence of anemia and hypoalbuminemia in this study. Preoperative hypoalbuminemia and comorbidities were reported to be independent risk factors for PPOI in several previous studies, indicating that the decreased nutritional and functional status may play a role in the development of PPOI. In our study, these factors were associated with PPOI in the univariate analysis (Table 1), but when they were included in the multivariate analysis, these associations became not significant, which can be explained by their connections with advanced age. This result suggested that advanced age can reflect a more comprehensive body functional and nutritional status, serving as an independent risk factor for PPOI.

In the present study, we identified postoperative body temperature as an independent risk factor for PPOI. The postoperative fever after gastric surgery is mostly absorbed heat which was caused by the absorption of aseptic necrotic substances and inflammatory factors, generally no more than $38.5^{\circ} \mathrm{C}$. Some fever was caused by postoperative infection, drug allergy and other reasons. Gastrointestinal tract is dominated by sympathetic nerve, parasympathetic nerve and enteric nerve. Sympathetic nerve usually inhibits gastrointestinal motility and gland secretion, while parasympathetic nerve regularly does the opposite. Fever may cause sympathetic nerve excitation, parasympathetic nerve inhibition and more water evaporation, thus causing gastrointestinal motility hypofunction, decreased secretion of digestive juice and decreased activity of digestive enzymes lead to anorexia, dry oral mucosa, abdominal distension and other clinical signs [15]. In this study, postoperative use of dezocine analgesia is also an independent risk factor for PPOI. Opiates have been widely reported to be independently associated with POI after colorectal surgery [16-18]. This conclusion was also confirmed in gastric cancer surgery by our study. It has been demonstrated that the inhibitory effect of opiates on postoperative gastrointestinal motility was mediated by peripheral $\mu$-opioid receptors. Postoperative opiates dose is one of the most important modifiable risk factors for POI. Therefore, various measures should be adopted to reduce the usage of opiates, including using nonsteroidal anti-inflammatory drugs as alternatives to opiate analgesics and using thoracic epidural analgesia.

In addition, it was reported that open surgery, previous abdominal surgery, hypoproteinemia, excessive infusion, perioperative blood transfusion, and delayed ambulation after operation were not conducive to the recovery of gastrointestinal function $[19,20]$. However, no significant statistical difference was found in the occurrence of PPOI among the above clinical indicators in this study. The reason may be related to the sample size.

There are several other limitations in this study. First, our current study is a single-center study. However, we optimized the study design to minimize possible bias. Second, there was a lack of robust external validation of the scoring system. Therefore, whether the proposed scoring system will retain its predictive capability in an independent dataset is yet to be determined. A prospective multiple-center study is required to provide evidence for the validation of the scoring system in the future.

\section{References}

1. Vather R, Bissett I (2013) Management of prolonged post-operative ileus: Evidencebased recommendations. ANZ J Surg 83: 319-324. [crossref]

2. Wolthuis AM, Bislenghi G, Lambrecht $\mathrm{M}$, fieuws $\mathrm{S}$, Buck van Overstraeten $\mathrm{A}$, et al (2017) Preoperative risk factors for prolonged postoperative ileus after colorectal resection. Int J Colorectal Dis 32: 883- 890. [crossref]

3. Vather R, Trivedi S, Bissett I (2013) Defining postoperative ileus: Results of a systematic review and global survey. J Gastrointest Surg 17: 962-972. [crossref]

4. Mao H, Milne T, O'Grady G, Vather R, Edlin R, et al. (2018) Prolonged postoperative ileus significantly increases the cost of inpatient stay for patients undergoing elective colorectal surgery: results of a multivariate analysis of prospective data at a single institution. Dis Colon Rectum 62: 631-637. [crossref]

5. Chapuis PH, Bokey L, Keshava A, Rickard MJFX, Stewart P, et al. (2013) Risk factors for prolonged ileus after resection of colorectal cancer: An observational study of 2400 consecutive patients. Ann Surg 257: 909- 915. [crossref] 
6. Huang DD, Zhuang CL, Wang SL, Pang WY, Lou N, et al. (2015) Prediction of prolonged postoperative ileus after radical gastrectomy for gastric. Medicine (Baltimore) 94: 2242. [crossref]

7. Moghadamyeghaneh Z, Hwang GS, Hanna MH, et al. (2016) Risk factors for prolonged ileus following colon surgery. Surg Endosc 30: 603-609.

8. Wolthuis AM, Bislenghi G, Fieuws S, de Buck van Overstraeten A, Boeckxstaens G, et al. (2016) Incidence of prolonged postoperative ileus after colorectal surgery: A systematic review and meta-analysis. Colorectal Dis 18: 1-9.

9. Kronberg U, Kiran RP, Soliman MSM, Hammel JP, Galway U, et al. (2011) A characterization offactors determining postoperative ileus after laparoscopic colectom enables the generation of a novel predictive score. Ann Surg 253: 78-81. [crossref]

10. Millan M, Biondo S, Fraccalvieri D, Frago R, Golda T, et al. (2012) Risk factors for prolonged postoperative ileus after colorectal cancer surgery. World J Surg 36: 179185. [crossref]

11. Dai X, Ge X, Yang J, Zhang T, Xie T, et al. (2016) Increased incidence of prolonged ileus after colectomy for inflammatory bowel diseases under ERAS protocol: a cohort analysis. J Surg Res 212: 86-93. [crossref]

12. Artinyan A, Nunoo-Mensah JW, Balasubramaniam S, Gauderman J, Essani R, et al. (2008) Prolonged postoperative ileus-definition, risk factors, and predictors after surgery. World J Surg 32: 1495-1500. [crossref]
13. Vather R, Josephson R, Jaung R, Kahokehr A, Sammour T, et al. (2015) Gastrografin in prolonged postoperative ileus: a double-blinded randomized controlled trial. Ann Surg 262: 23-30. [crossref]

14. Hain E, Maggiori L, Mongin C, Prost AlDJ, Panis Y (2018) Risk factors for prolonged postoperative ileus after laparoscopic sphincter-saving total mesorectal excision for rectal cancer: An analysis of 428 consecutive patients. Surg Endosc 32: 337-344. [crossref]

15. Wolthuis AM, Bislenghi G, Fieuws S, Overstraeten AB, Boeckxstaens G, et al. (2016) Incidence of prolonged postoperative ileus after colorectal surgery: A systematic. Colorectal Dis 18: 1-9.

16. Bragg D, El-Sharkawy AM, Psaltis E, Maxwell-Armstrong CA, Lobo DN, et al. (2015) Postoperative ileus: Recent developments in pathophysiology and management. Clin Nutr 34: 367-376. [crossref]

17. Thomas J (2008) Opioid-induced bowel dysfunction. J Pain Symptom Manage 35: 103-113.

18. Wood JD, Galligan JJ (2004) Function of opioids in the enteric nervous system. Neurogastroenterol Motil 16: 17-28.

19. Kibler VA, Hayes RM, Johnson DE, Anderson LW, Just SL, et al. (2012) Cultivating quality: early postoperative ambulation: Backtobasics. Am J Nurs 112: 63-69. [crossref]

20. Brandstrup B (2006) Fluid therapy for the surgical patient. Best Pract Res Clin Anaesthesiol 20: 265-283. [crossref]

\section{Citation:}

Zheng Yiqiong (2021) Incidence and Factors of Prolonged postoperative Ileus in Gastric Cancer Surgery. Cancer Stud Ther J Volume 6(1): 1-4. 